\title{
Cognitive dysfunction in corticobasal degeneration
}

\author{
Disfunção cognitiva na degeneração corticobasal \\ Lais Machado de Oliveira', Igor Barcellos², Hélio A.G. Teive³, Renato Puppi Munhoz'
}

\begin{abstract}
Corticobasal degeneration (CBD) was originally described as a distinct clinicopathological entity in 1967. Since then, different phenotypic presentations have emerged as possible manifestations of CBD histopathological findings. In addition, pathophysiological findings and the molecular basis have been delineated and several aspects of its cognitive manifestations have been clarified. Thus, not only the spectrum of what is currently designated as CBD has expanded, but overlap with other degenerative and even secondary disorders has made clinical diagnostic certainty even more challenging in the absence of specific and readily-available markers. Cognitive deficits in CBD are now recognized as a frequent initial presentation and may appear up to eight years before the motor symptoms, depending on the phenotypic variant. Characteristic cognitive features of CBD involve language deficits, visuospatial and executive dysfunctions, apraxia, and behavioral disorders. Semantic and episodic memories are usually preserved, while language is often impaired in the early stages.
\end{abstract}

Keywords: dementia; cognition; corticobasal degeneration.

\section{RESUMO}

A degeneração corticobasal (DCB) foi originalmente descrita como uma entidade clínico-patológica distinta em 1967. Desde então, nossa compreensão sobre DCB evoluiu substancialmente. Diferentes apresentações fenotípicas emergiram refletindo possíveis manifestações das anormalidades histopatológicos da DCB. Adicionalmente, dados fisiopatológicos e moleculares foram delineados e aspectos das manifestações cognitivas foram explorados. Assim, não só o espectro do que é atualmente designado DCB foi expandido, mas a sobreposição com outras doenças degenerativas e até mesmo secundárias tornaram o diagnóstico clínico ainda mais desafiador na ausência de marcadores específicos e prontamente disponíveis. Déficits cognitivos na DCB são agora reconhecidos frequentemente como apresentações iniciais e podem surgir até 8 anos antes dos sintomas motores, dependendo da variante fenotípica. 0 quadro cognitivo envolve característicamente déficits de linguagem, disfunção visuoespacial e executiva, apraxia, e distúrbios comportamentais. Anormalidades da linguagem são frequentemente descritas nos estágios iniciais da DCB.

Palavras-chave: demência; cognição; degeneração corticobasal.

Corticobasal degeneration (CBD) is a rare and progressive neurodegenerative disease. It was originally described as a distinct clinicopathological entity in $1967^{1}$ and later recognized as a polymorphous disease due to its complexity. In fact, the classical set of symptoms - namely asymmetric motor features associated with higher cortical function - were found to be associated with multiple histological presentations. Furthermore, the distinctive histopathological findings of cortical and striatal neuronal deposition of tau, and glial inclusions, were also linked to a wide variety of clinical phenotypes ${ }^{2}$. Hence, recent literature refers to corticobasal syndrome (CBS) as the constellation of symptoms described originally, limiting CBD to the distinctive histopathological findings ${ }^{3}$.

The different terminology with a seemingly paradoxical intrinsic overlap between these conditions makes diagnosis challenging. For instance, only $25 \%$ to $56.25 \%$ of patients with pathologically-confirmed CBD had previously presented with symptoms of $\mathrm{CBS}^{4,5,6}$. On the other hand, the proportion of patients diagnosed with CBS presenting with the histologic hallmarks of CBD is highly variable, ranging from $23.8 \%$ to $100 \%{ }^{4,5,6,7}$. Considering the enormous difficulty in accurately diagnosing CBD during life, it is clear that current

\footnotetext{
${ }^{1}$ University Health Network, Toronto Western Hospital, Morton and Gloria Shulman Movement Disorders Centre and the Edmond J. Safra Program in Parkinson's Disease, Toronto, ON, M5T 2S8, Canada.

2 Pontifícia Universidade Católica do Paraná, Hospital Universitário Cajuru, Serviço de Neurologia, Curitiba PR, Brasil;

${ }^{3}$ Universidade Federal do Paraná, Hospital de Clínicas, Departamento de Medicina Interna, Serviço de Neurologia, Setor de Distúrbios do Movimento, Curitiba, PR, Brasil.
}

Correspondence: Renato Puppi Munhoz; University of Toronto, Movement Disorders Centre, Toronto Western Hospital; 399 Bathurst St, 7 Mc412, Toronto, ON Canada M5T 2S8; E-mail: renato.munhoz@uhn.ca

Conflict of interest: There is no conflict of interest to declare.

Received 28 January 2017; Accepted 30 March 2017. 
epidemiological estimates hold substantial uncertainty. As a matter of fact, there are no estimates for CBD. Regarding CBS, a community-based Japanese study found an ageadjusted prevalence rate of 6 per $100,000^{8}$, while a Russian study in a large district of Moscow showed an age-standardized incidence of CBS of 0.02 per 100,000 person-years9. Aiming at improving an antemortem diagnosis, new clinical criteria were developed for CBD diagnosis, but await validation $^{10}$. These new criteria have expanded the recognized clinical phenotypes, reflecting current knowledge. Still, it seems to lack specificity for the clinical diagnosis ${ }^{11}$ and sensitivity within the first two years of disease onset?

The disease typically manifests in the sixth to seventh decades. Mean age of onset is 63.7 (ranging from 45 to 77.2) years and mean duration of disease is six to seven years ${ }^{12}$, with shorter survival in those patients who initially present with dementia ${ }^{13}$. There is no strong evidence for gender propensity and no established environmental risk factors ${ }^{13}$. Finally, there is also no effective specific therapy for $\mathrm{CDB}^{12,14}$.

The aim of this review was to provide a comprehensive review of the clinical features of CBD, focusing on the cognitive aspects of the disease. We will also try to correlate these findings, and explore the mechanisms, particularly the distribution of tau pathology in the central nervous system (CNS).

\section{The clinical spectrum associated with corticobasal degeneration}

CBD has typically been associated with different combinations of asymmetric motor symptoms (parkinsonism, dystonia and myoclonus), and higher cortical dysfunction (ideomotor apraxia, alien-limb phenomena and cortical sensory loss). However, a wide range of clinical presentations beyond the archetypal CBS-related phenotype have emerged as possible manifestations of CBD pathology, including the nonfluent/agrammatic variant of primary progressive aphasia, frontal behavioral-spatial syndrome, and progressive supranuclear palsy (PSP-CBD) $)^{15}$. Less common manifestations include posterior cortical atrophy and dementia with features of Alzheimer's disease (AD) $)^{13}$.

In a recent review of pathologically-proven $\mathrm{CBD}$, limb rigidity (85\%) and bradykinesia (76\%) were the most common motor findings, usually associated with absent or transient levodopa responsiveness ${ }^{16}$. Limb rigidity might reflect a combination of parkinsonism, dystonia and paratonia. Postural instability, falls and abnormal gait were reported in $78 \%, 75 \%$ and $73 \%$ of patients respectively ${ }^{16}$.

Limb dystonia was frequently reported in CBS and mixed CBS/CBD series ${ }^{17,18}$, but was present in only $38 \%$ of CBD patients during the disease course. Another study of pathologically-proven CBD reviewed the presence of dystonia in 196 cases and showed similar results $(37.5 \%)^{19}$. Unilateral upper limb dystonia is the most common distribution and may progress to hemidystonia or affect the contralateral side. Blepharospasm and axial dystonia may occur (usually not in isolation) and are more frequent in the PSP-CBD phenotype ${ }^{2}$. Patients with CBD who present with prominent cognitive features - typically frontotemporal dementia-CBD and AD-CBD phenotypes - might also develop dystonia, typically later in the course of the disease when compared to the CBS-CBD phenotype ${ }^{19}$.

Myoclonus is a frequent finding in mixed CBS-CBD series $^{18,20}$ but was only present in $27 \%$ of the patients with CBD. It predominantly affects the upper extremities ${ }^{13}$ and can be movement and/or stimulus sensitive. Due to its focal and stimulus-sensitive presentation, a cortical origin was suggested. Interestingly, electrophysiological findings in CBS patients with myoclonus seem to differ from the known cortical reflex myoclonus: shorter latency is observed and there is no enlargement of the secondary component of the cortical somatosensory evoked potential, nor association with cortical spikes on back-averaging ${ }^{21}$. It is unknown whether CBD presents with the same electrophysiological features.

Tremor occurs in $39 \%$ of the patients and may manifest with rest, postural and action components. Distinction from low-amplitude myoclonus can be challenging ${ }^{21}$. Abnormal eye movements were reported in $60 \%$ of CDB patients, but insufficient details were provided. Increased saccadic latency has been described in $\mathrm{CBS}^{22,23}$, but analysis of CBD patients failed to demonstrate this ${ }^{24}$.

Cognitive impairment during the course of the disease occurs in $70 \%$ of cases and $52 \%$ had this symptom at presentation $^{25}$. Cognitive dysfunctions in CBD will be discussed in detail, further in this review.

\section{Neuropathology and pathogenic mechanisms}

Tau protein binds to microtubules and promotes polymerization. This protein is encoded by the microtubule associated tau protein (MAPT) gene, of which alternative splicing results in six different isoforms. The MAPT is highly expressed in neuronal cells, and at lower levels in glial cells. Tau is essential for microtubule stability, axonal transport, synaptic health and neuronal integrity, among other roles. Hyperphosphorylation of tau is a post-translational change that has important functional roles in the binding affinity to microtubules. Furthermore, dissociated tau is prone to multimerization, accumulating in the cytoplasm in the form of intracellular inclusions ${ }^{26}$. The processes that induce tau phosphorylation are currently unknown but might involve microglia signaling triggered by neuroinflammation ${ }^{27}$. Additionally, neurodegeneration mechanisms elicited by tauopathies are still unclear and might be related to synaptic dysfunctions ${ }^{28}$. Morphological analysis with characterization of the cell types and anatomical areas involved, along with evaluation of different tau isoforms (reflecting biochemical heterogeneity) are key steps to distinguishing among different tauopathies.

Macroscopic examination of CBD shows asymmetrical cortical atrophy, predominantly in the perirolandic area, posterior-frontal to the parietal area, anterior frontal, or in the 
perisylvian area. Severe depigmentation of the substantia nigra is also a frequent gross finding ${ }^{26,29,30}$. The characteristic microscopic features of CBD are cortical and striatal tau-related neuronal inclusions, accompanied by astrocytic plaques. Ancillary histologic techniques, such as the Gallyas silver staining and tau immunohistochemistry, help in identifying the plaques and neuronal inclusions. Thread-like lesions are also generally present. Achromatic or ballooned cortical neurons, described by Rebelz, are relevant but not specific for CBD diagnosis ${ }^{28,29,30}$.

Corticobasal degeneration and PSP share similar morphological features, including diffuse neuronal cytoplasmic tau-immunoreactivity, threads in the cortex, white and gray matter, and oligodendroglial coiled bodies. Furthermore, isoform-specific monoclonal antibodies reveal that both conditions are predominantly $4 \mathrm{R}$ tauopathies. However, differential diagnosis can be achieved based on other histological findings and their anatomical distribution. For instance, the main microscopic features of PSP involve neurofibrillary tangles and threads, particularly in the subthalamic nucleus, basal ganglia and brainstem, and the glial lesions are mainly characterized by tufted astrocytes. The distinct astroglial tau-pathology and the predominant involvement of the forebrain in CBD and the hindbrain in PSP help to distinguish these two conditions ${ }^{31}$.

Immunoblotting may also help to differentiate between these disorders. Although the patterns of insoluble tau observed in CBD and PSP are bands at 64 and $68 \mathrm{kDa}$, the cleaved tau fragments from patients with CBD migrate as two bands (a doublet of around $37 \mathrm{kDa}$ ), whereas those from patients with PSP migrate as a single band of $33 \mathrm{kDa}^{28}$.

Finally, along with morphological and biochemical studies, the genetic basis of tauopathies is also relevant. The $\mathrm{H} 1$ haplotype and the Hlc sub-haplotype of the MAPT gene are overrepresented in CBD and PSP, and are thus considered possible risk factors ${ }^{32}$. In addition, genome-wide studies have looked at other potential susceptibility loci, such as 3p22 myelin-associated oligodendrocyte basic protein ${ }^{31}$. Despite some evidence for genetic predisposition, CBD is largely a sporadic disorder, although there are rare familial cases involving mutations in the MAPT gene and possibly MRS2 and ZHX2 genes ${ }^{33}$.

\section{New approaches and potential biomarkers}

As postmortem examination remains the gold standard for CBD diagnosis, patients and clinicians face growing anxiety and uncertainty. Therefore, reliable biomarkers would be valuable tools ${ }^{15}$. In addition, as studies on tau-directed therapies advance, there will be an increasing need for surrogate markers that can monitor the effect of such therapeutic interventions ${ }^{34}$. From both perspectives, improvement in imaging techniques and cerebrospinal fluid protein analysis is critical.

Currently, there is no effective imaging method to confidently differentiate CBS-CBD from other tauopathies, particularly PSP. In a voxel-based morphometry study comparing atrophy patterns in CBD and PSP, the former displayed higher cortical gray matter atrophy affecting the posterior frontal and parietal regions, moderate subcortical gray matter atrophy and substantial basal ganglia atrophy, with relatively-spared brain$\mathrm{stem}^{35}$. A volumetric study using three-dimensional magnetic resonance showed significant reductions in average brain, brainstem, midbrain, and frontal gray matter volumes in PSP, while CBD patients presented with parietal cortex and corpus callosum atrophy. Moreover, the authors stated that the model using the combination of midbrain, parietal white matter, temporal gray matter, brainstem, frontal white matter and pons volumes had a high degree of accuracy in differentiating PSP, CBD and healthy control patients ${ }^{36}$. Another longitudinal MRI study in five CBD patients found that this disorder had the largest rate of whole brain atrophy compared to PSP and other neurodegenerative disorders ${ }^{37}$. Finally, diffusion tensor imaging (DTI) studies in CBS patients consistently showed diffuse white matter abnormalities ${ }^{38,39}$, and a more recent longitudinal DTI study comparing CBS and PSP patients at baseline and six-month follow-up showed that CBS patients had higher DTI changes in the pre- and post-central, superior parieto-occipital and temporal white matter during the study period than PSP patients ${ }^{34}$.

The assessment of metabolic changes using FDG-PET scanning may also be helpful. Although asymmetric metabolic reductions involving frontal and parietal cortex, thalamus, and caudate nucleus are not specific for CBD diagno$\mathrm{sis}^{40}$, one study hypothesized that parietal hypometabolism may be a distinctive feature ${ }^{41}$. Additionally, targeted methods such as SPECT and PET assessing striatal dopaminergic activity, and techniques focused on specific markers, such as tau protein, are of growing interest. A study using [123I] IBZM SPECT showed variable findings regarding striatal D2 receptor binding in autopsy-proven CBD cases $^{42}$, but a subsequent study from the same group stressed the importance of follow-up imaging in suspected cases ${ }^{43}$. Imaging pathological tau using PET is desirable, but the development of an appropriate tracer is challenging, as it should be able to cross the cell membrane and bind to phosphorylated tau in neuronal and glial cells. In one study, the [11C]-PBB3 ligand was able to detect intracellular tau in $\mathrm{AD}$ and non- $\mathrm{AD}$ tauopathies in transgenic mouse models and in humans, including pathological tau within neurons and astrocytic plaques in $\mathrm{CBD}^{44}$. A single-patient study assessing [18F]AV-1451 PET uptake correlated the tracer uptake with underlying tau burden at autopsy ${ }^{45}$. These results are promising, but further and larger studies are needed to better validate their findings.

Besides imaging techniques, cerebrospinal fluid protein analyses are expected to effectively improve antemortem diagnoses of tauopathies. The assessment of tau and phosphorylated tau are useful biomarkers in recently-established $\mathrm{AD}$, but have yielded varied results in $\mathrm{CBD}^{13}$. Moreover, a study analyzing tau fragments ( $\mathrm{N}$-terminal and $\mathrm{C}$-terminal) concentrations found lower levels in PSP patients than in AD and healthy controls ${ }^{46}$, but these preliminary results need to be confirmed in pathologically-proven PSP. Additional studies in CBD are needed. 


\section{Main cognitive features of corticobasal degeneration}

Recent studies have revisited the role of dementia in CBD diagnosis criteria ${ }^{2,47}$. Past criteria had excluded early dementia from the CBD clinical picture, but advances in our understanding of the phenotypic presentations of CBD have contributed to include dementia back in the core of the $\mathrm{CBD}$ criteria discussion².

Data on the proportion of higher cortical features in CBD patients were defined (a) at presentation and (b) during the disease course ${ }^{2}$ :

1) Cognitive impairment - 52\% (a) and $70 \%$ (b)

2) Behavioral changes $-46 \%$ (a) and $55 \%$ (b)

3) Limb apraxia - $45 \%$ (a) and $81 \%$ (b)

4) Aphasia - 40\% (a) and $52 \%$ (b)

5) Depression $26 \%$ (a) and $51 \%$ (b)

6) Cortical sensory loss - $25 \%$ (a) and $27 \%$ (b)

7) Alien limb phenomena - 22\% (a) and 30\% (b).

Previous studies have already highlighted the importance and burden of cognitive symptoms in $\mathrm{CBD}^{5}$. Furthermore, in addition to the classic asymmetrical movement disorder with lateralized higher cortical features, CBD patients display a distinctive cognitive profile pattern that must be faced as a core feature of the clinical entity.

The CBD cognitive deficits are related to the predominantly-affected cerebral hemisphere and include apraxia, visuospatial dysfunction, language impairment, executive dysfunction and behavioral changes with relatively preserved episodic and semantic memories that will be discussed in detail below ${ }^{2,47}$.

\section{Apraxia}

Classically, apraxia can be the most-recognizable and exuberant cognitive deficit in CBD. Ideomotor apraxia is the mostcommonly described (57\%), followed by limb-kinetic apraxia. Both are related to the action production system ${ }^{248}$, while ideational apraxia is related to the conceptual motor system. The observation that left hemispheric lesions tend to result in bilateral upper limb apraxia has suggested that movement memory is stored in the left parietal cortex as a "space-time picture" that may be accessed by both frontal lobes, where the motor engrams are located. For instance, if voluntary movement is performed with the left upper limb, the information about what to do must first pass throughout the corpus callosum to access the contralateral hemisphere ${ }^{48,49}$.

Apraxia is often asymmetrical. When presenting primarily in the dominant hand, it usually manifests as an ideomotor disorder for meaningful movements. Alternatively, the presentation in the nondominant hand often includes spatial errors during reproduction of a gesture relating to different body parts².

As an action production system deficit, ideomotor apraxia causes difficulties using tools or mimicking tool use. Also, patients commit errors in scaling, timing and orientation of movements ${ }^{48}$. Here, the idea of movement is intact but there is a difficulty in mapping motor memory traced in the frontal lobes ${ }^{48}$. Patients tend to improve performance when possessing the real object that was part of the action they were asked to pantomime ${ }^{48}$. As the recognition of an action goal is usually preserved, ideomotor apraxia is usually less disabling than ideational apraxia.

Limb-kinetic apraxia is characterized by impairment in the production of fine motor movements due to disruption in the final stage of motor engrams in the prefrontal cortex ${ }^{48}$. Previously, this type of apraxia was mistakenly considered a pure dexterity problem, but this concept has been reviewed due its high prevalence in $\mathrm{CBD}^{48}$. Limb-kinetic apraxia can be assessed with finger-tap maneuvers as well as asking the patient to oppose their thumb to their index, middle, ring and little fingers quickly back and forth ${ }^{48}$. It can also coexist with ideational and ideomotor apraxia in CBD.

Ideational apraxia consists of a deficit in the conceptualization of an action and the idea of movement itself $f^{48,49}$. Patients usually cannot understand how and why to use a tool although they can name it correctly. Also, they are unable to describe how to perform an action and pantomime it. Ideational apraxia is less common than ideomotor and limb-kinetic apraxia in CBD patients.

Constructional apraxia and handwriting impairment also are common in CBD. They are evident in drawing tests, copying figures or even writing. Difficulties defining shape and letters can also be present. This finding can be an early sign of cognitive impairment in $\mathrm{CBD}^{50}$. Spelling impairment is uncommon.

Apraxia of speech is the disruption between neural representation of a grammatical sentence and orofacial muscular activity $^{48}$. Slowness and effortful speech, as well as difficulties in ordering and timing syllables, are characteristic. Changes in the prosody of speech and intonation are also common. Buccofacial apraxia is the inability to perform non-speech-related movements involving the face and tongue muscles. Apraxia of eyelid opening is a transient difficulty opening the eyes usually due to impairment of the supranuclear control of eyelid elevation. Oculomotor apraxia is an inability to make voluntary saccades to a certain visual target ${ }^{48}$. Agraphesthesia and astereognosis may also be associated with apraxia.

\section{Language and speech}

Aphasia is an early cognitive symptom present in $40 \%$ of $\mathrm{CBD}$ patients at the first assessment $t^{2}$. The spectrum of language impairment can range from slight dysphonia to severe aphasia ${ }^{50}$. Agrammatic or nonfluent aphasia is the most common language presentation of CBS, especially in the nonfluent/agrammatic primary progressive aphasia-CBD phenotype ${ }^{2}$.

Cognitive-onset CBD patients present with aphasia earlier than motor-onset CBD patients, although most of them develop some degree of language impairment during disease progression $^{50}$. Initially, phonologic handwriting and spelling 
impairment tend to be more common. Naming is usually unaffected or mildly impaired in the early stages. In addition, most of the patients exhibit poor performances in category and letter fluency tests ${ }^{50}$.

Progressive nonfluent aphasia is characterized by disturbances in the motor component of language, sparing the semantic component, as are apraxia of speech and agrammatism $^{51}$. This cognitive syndrome can be present in a bunch of tauopathies, classically in fronto-temporal dementia, but also in $\mathrm{AD}^{52}$. Beyond nonfluent aphasia, patients may also experience marked reduction of speed fluency and grammar deficits ${ }^{52}$.

Language evaluation comprises naming, comprehension, repetition, reading and writing. Single-word processing can be assessed with confrontation naming and is usually impaired in the nonfluent/agrammatic variant of primary progressive aphasia. Comprehension can be assessed by providing written and verbal command, asking the patient to write a sentence and repeat words and sentences and, finally, asking the patient to read some words or a sentence ${ }^{53}$.

Of note, a more generalized pattern of atrophy involving the inferior frontal and temporal lobes was related to CBS patients presenting with marked dementia and aphasia ${ }^{50}$.

\section{Visuospatial abilities}

It is well established that CBD patients have a marked visuospatial dysfunction ${ }^{52,54}$. Like language dysfunction, visuospatial deficits also present as a spectrum of symptoms, including Balint's syndrome. The rate of impairment in this cognitive domain ranged from $28 \%$ to $52 \%$ in CBD patients, depending on the test used ${ }^{54}$.

Visuospatial abilities can be assessed by asking the patient to draw two interlocking pentagons (or other geometric figures like a cube, a circle, two overlapping rectangles) or performing the clock face-drawing test. The Rey-Osterreith Complex Figure test can also be used ${ }^{53,54}$. In fact, CBD patients may present with other cognitive and motor deficits, such as apraxia, executive dysfunction and dystonia, which may negatively affect the neuropsychological exam of visuospatial abilities.

As CBD pathology involves dorsal occipitoparietal cortex, patients often perform worse on spatial location tasks, probably due to parietal atrophy affecting the dorsal stream of visuospatial processing ${ }^{11}$. The planning component of visual construction also can be impaired, overlapping with executive dysfunction due to frontal pathology.

\section{Executive function}

Executive function refers to the ability of planning, judging, reasoning, problem solving, and to organization, abstraction, and mental flexibility ${ }^{55}$. Executive dysfunction in CBD patients has been reported consistently in the literature ${ }^{2}$.

In a study of $50 \mathrm{CBS}$ patients and 51 fronto-temporal dementia patients, using the Delis-Kaplan Executive Function System $^{55}$, executive function was better preserved in patients with CBS than in fronto-temporal dementia patients, except for tests that required visuospatial and motor abilities ${ }^{55}$. Both groups showed poorer executive function performance than memory. Impaired planning, limited mental search and poor inhibitory control were documented in CBS patients, although caregivers rarely complained of executive difficulties. Early verbal fluency impairment has been reported in previous studies ${ }^{2,55}$.

No studies had correlated executive function and pathology in CBD. In one CBS study, executive dysfunction was associated with dorsal frontal and temporal-parietal cortex volume loss in voxel-based morphometry ${ }^{55}$.

The verbal fluency (letter and category), sorting test, tower test, and trail making test are effective ways to assess executive dysfunction. The Wisconsin Card Sorting Test, as well as the Delis-Kaplan Executive Function System, are also used.

\section{Memory}

Both semantic and episodic memory seem to be mildly impaired or relatively preserved in $\mathrm{CBD}^{50}$. Although $\mathrm{CBD}$ patients' performance in memory tests were variable in different studies, they tended to perform better than $\mathrm{AD}$ patients. Furthermore, atrophy is concentrated mainly in the frontal cortex rather than the hippocampus in CBD. In one study, 15 CBS patients had performed better on story recall and list recall tests than a group of $\mathrm{AD}$ patients ${ }^{55}$. It is believed that episodic memory impairment in CBD is due to frontal lobe dysfunction, reflecting a poor use of encoding and retrieval strategic processes ${ }^{55}$. Over and above, findings regarding episodic memory in CBD are inconsistent. The few studies that evaluated semantic memory suggest that this cognitive domain is relatively preserved in CBD patients.

\section{Behavioral dysfunction}

Social behavior is occasionally impaired in CBD patients, especially in the frontal behavioral-spatial syndrome variant ${ }^{2}$. Relatives and caregivers usually report disinhibition, aggressiveness, perseveration, hypersexuality, bizarre behavior, hyperorality, and unmotivated laughter ${ }^{2}$. Insight is often absent or dramatically impaired in these patients ${ }^{56}$. On the other hand, apathy is frequently part of the CBD clinical picture. Depression may be a symptom of CBD, although its frequency was not different from the control group in a cohort study².

\section{Cognitive assessment in corticobasal degeneration}

Previous studies and reviews have approached cognitive symptoms in CBD, proposing a wide variety of cognitive tests (Table), but none of them in a systematic and objective way ${ }^{57}$.

Recently, two studies proposed the Addenbrooke's Cognitive Examination Revised (ACE-R) for both objective screening and cognitive diagnosis of $\mathrm{CBD}$ and other parkinsonian syndromes ${ }^{51,52}$. This test combines the agility of the Mini-Mental State Examination, with a better evaluation of memory, language, visuospatial cognition and verbal fluency. 


\begin{tabular}{|c|c|c|}
\hline Cognitive domain & Characteristics and presentation & Assessment \\
\hline \multirow{7}{*}{ Apraxia* } & Ideomotor apraxia & Luria's three-step task \\
\hline & Limb-kinetic apraxia & Pantomiming actions \\
\hline & Ideational apraxia & Meaningful and meaningless hand gestures \\
\hline & Constructional apraxia & "Lick your lips / Cough” \\
\hline & Buccofacial apraxia & "Open your eyes" \\
\hline & Apraxia of eyelid opening & Ocular movements examination \\
\hline & Oculomotor apraxia & \\
\hline \multirow{3}{*}{ Visuospatial ability } & Poor spatial localization sense & $\begin{array}{l}\text { Copying: two interlocking pentagons; Rey- } \\
\text { Osterreith Complex Figure test; a cube; a circle; } \\
\text { two overlapping rectangles; a lozenge. }\end{array}$ \\
\hline & Visual construction impairment (planning component) & Clock face drawing test \\
\hline & Balint's syndrome & \\
\hline \multirow{7}{*}{ Language } & Dysphonia & Verbal Fluency Tests (letter and category) \\
\hline & Phonologic handwriting and spelling impairment & Confrontation naming \\
\hline & Naming difficulties & Boston Naming test \\
\hline & $\begin{array}{l}\text { Nonfluent aphasia (motor component of language, speech } \\
\text { apraxia and agrammatism) }\end{array}$ & Repetition \\
\hline & & Comprehension (multistep tasks) \\
\hline & & Reading \\
\hline & & Writing (dictation and spontaneous sentences) \\
\hline \multirow{6}{*}{ Executive function } & Working memory, inhibition and set shifting impairment & Verbal Fluency Tests \\
\hline & $\begin{array}{l}\text { Difficulties in planning, judging, reasoning, problem solving, } \\
\text { abstraction and mental flexibility }\end{array}$ & Sorting Test \\
\hline & & Tower Test \\
\hline & & Trail Making Test \\
\hline & & Wisconsin Card Sorting Test \\
\hline & & D-KEFS ${ }^{a}$ \\
\hline \multirow{4}{*}{ Behavior } & $\begin{array}{l}\text { Disinhibition, aggressiveness, perseveration, hypersexuality, } \\
\text { bizarre behavior, hyperorality, and unmotivated laughter }\end{array}$ & History referred by patient's relatives \\
\hline & Impaired or absent insight & BDI-II ; HAM-D; MADRS \\
\hline & Apathy & Raskin Depression Rating Scale; IDS ${ }^{e}$; GDS ${ }^{f}$ \\
\hline & Depression (less common) & \\
\hline
\end{tabular}

Additionally, the ACE-R was able to detect early stages of dementia. In one study, the ACE-R detected cognitive dysfunction in $90 \%$ of CBS patients with $91 \%$ sensitivity and $98 \%$ specificity using a cutoff of 88 to 89 . In comparison with progressive nonfluent aphasia, CBS showed a similar cognitive impairment profile, except for poorer visuospatial function in $\mathrm{CBS}^{52}$. Another study evaluating the ACE-R in 135 patients with parkinsonian syndromes (86 PD; 30 PSP; 19 CBD) $)^{51}$ proposed that the verbal fluency subscore was an objective contributor to differentiate parkinsonian syndromes. Data analysis suggested that the ACE-R can be useful for tracking cognitive CBD progression over the time ${ }^{51}$.

Apraxia can be assessed with a sequence of commands and gestures as proposed first by Green ${ }^{57}$ and then improved by
Cassidy ${ }^{48}$. Examination starts with asking the patient to mimic a certain hand gesture as well as asking how to use a tool to perform a trivial action. Buccofacial apraxia is assessed by asking the patient to lick their lips or cough. Lurias three-step task is commonly used, as well as hand gestures such as the "okay sign" to verify ideomotor apraxia ${ }^{48}$ (Figure).

Thus, the ACE-R and apraxia sequence evaluation together could provide an accurate and quick cognition assessment in CBD patients.

\section{Relationship between motor and cognitive abnormalities}

Trying to define a chronological correlation between the onset of motor and cognitive symptoms in CBD patients is 

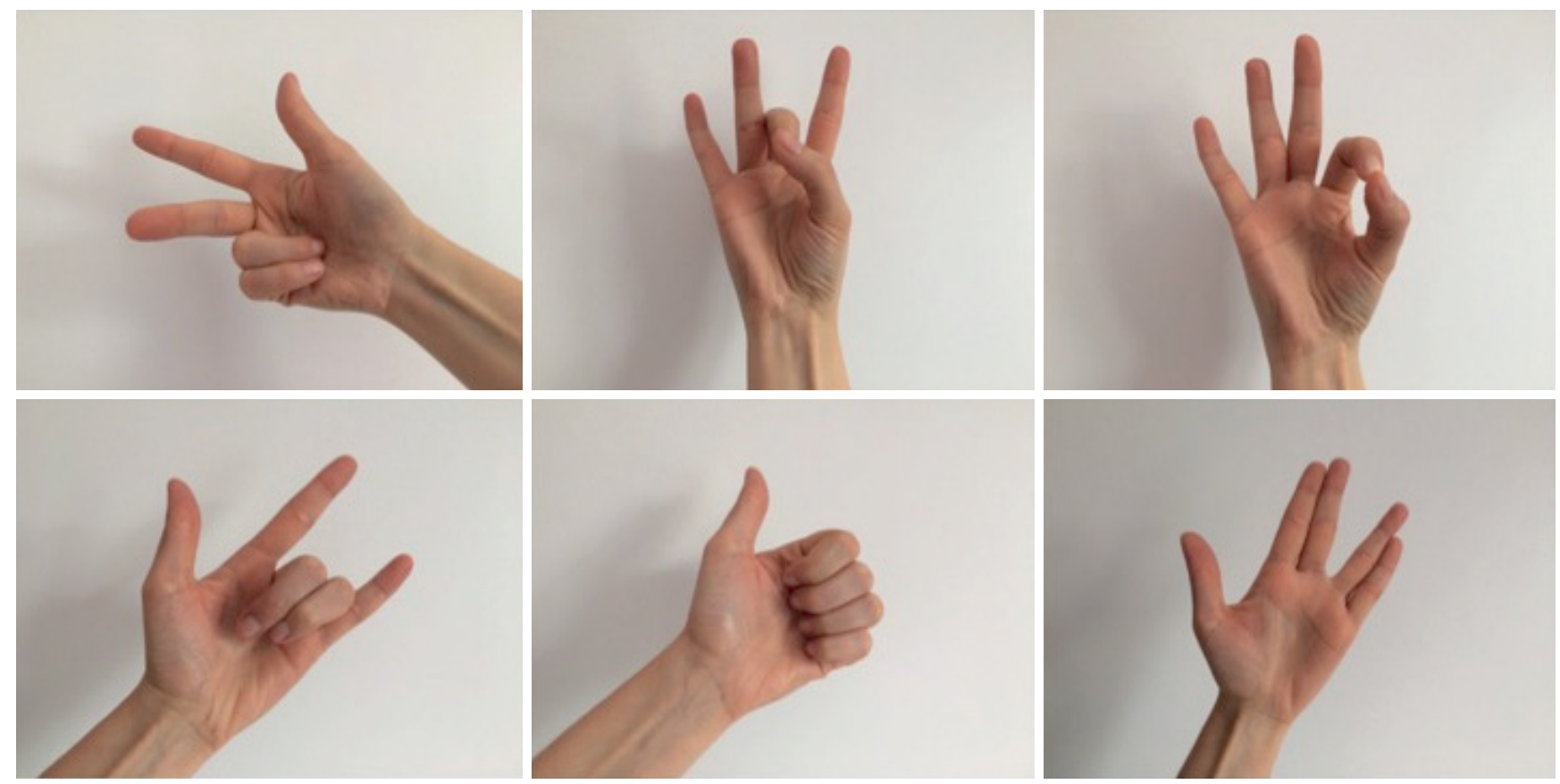

Figure. Gestures that can be used in apraxia assessment. The patient is asked to mimic gestures performed by the examiner.

difficult. The great variability of clinical features and different variants and phenotypes make this task even more challenging.

The first cohort study to assess the natural history in CBD found an average disease duration of 7.9 years $^{2}$. Few patients presented at first evaluation with early cognitive symptoms such as memory loss, aphasia, ideomotor apraxia and frontal lobe behavior. The most common initial manifestations were limb clumsiness $(50 \%)$, gait disorder (36\%), falls (21\%), unilateral painful paresthesia (29\%), frontal lobe symptoms (21\%) and dysarthria (14\%). At initial assessment, on average three years after onset, the patients presented mainly with unilateral or asymmetric limb bradykinesia or rigidity (78\%) as well as ideomotor apraxia (64\%). In fact, early CBD descriptions considered dementia a late, or even nonexistent, feature ${ }^{2}$.

However, later studies consistently suggested that dementia is an early symptom of CBD, sometimes presenting even before the onset of movement disorders ${ }^{2}$. A cohort followed 15 patients with pathological confirmation of $\mathrm{CBD}$, evaluating motor and cognitive symptoms during the disease progression. The rate of cognitive decline in these patients was 3 points per year in the MiniMental State Examination ${ }^{2}$. At presentation, gait disturbance was the most frequent motor complaint with unilateral rigidity or axial rigidity on examination. Involuntary movements such as myoclonus, dystonia and alien hand phenomena were observed at presentation in $20 \%$ of the patients and any of these signs were present in one-third of the cohort at death ${ }^{2}$.

Apraxia and visuospatial deficits were evidenced at examination in almost half of the patients at some point of disease, and may occur any time during disease course. Language dysfunction was the most common complaint in early CBD, even before motor symptoms. Patients experienced difficulties in word finding, handwriting, and effortful speech at this stage. With disease progression, naming, language output and even mutism were noted at examination. Language comprehension was initially preserved and was impaired only at the final stage of disease. Although it is not an early cognitive sign, all patients of the cohort had developed some level of executive dysfunction at some point prior to death'.

Another cohort study with 18 patients evaluated cognitive and motor symptoms throughout the study period, considering four clinical syndromes associated with CBD: five patients with progressive nonfluent aphasia, five with behavioral variant frontotemporal dementia, seven with executivemotor, and only one patient with posterior cortical atrophy ${ }^{58}$. Fifteen of the 18 patients had behavioral or cognitive impairment as the initial symptom, while less than half presented with motor finding ${ }^{58}$.

The first symptoms for CBD-progressive nonfluent aphasia were speech or language difficulties followed by motor symptoms one to five years later. Social withdrawal was the most common first behavioral symptom in behavioral variant-fronto-temporal lobar dementia-CBD, progressing to motor symptoms, mainly gait impairment, only after two to eight years. In the executive-motor-CBD group, three patients presented with early cognitive or behavioral and motor symptoms coincidently. The only patient with posterior cortical atrophy-CBD presented with reading complaints, and developed right hand apraxia two years later. 
At the last visit, all groups had higher rates of motor signs, especially the executive-motor-CBD group ${ }^{58}$.

\section{Uncommon variants of corticobasal degeneration}

Recently, the diagnostic criteria of CBD were redefined ${ }^{2}$. Five different phenotypes were identified: probable and possible CBS, frontal behavioral-spatial syndrome variant, progressive nonfluent aphasia variant, and PSP syndrome ${ }^{2}$. Although $55 \%$ of CBD patients present with the classic clinical picture, identification of the three other phenotypes are also of major importance ${ }^{12}$.

Besides motor symptoms, the diagnosis of frontal behavioral-spatial syndrome-CBD requires at least two of these three cognitive features: executive dysfunction; behavioral or personality changes; or visuospatial deficits ${ }^{2}$.

Language dysfunction is the core cognitive feature in progressive nonfluent aphasia-CBD. The diagnosis of the latter requires effortful and agrammatic speech plus at least one of these two features: impaired grammar or sentence comprehension with relative single word comprehension; or apraxia of speech ${ }^{2}$. In a recent study, these patients showed greater longitudinal changes in prefrontal anterior, medial, and lateral gray and white matters ${ }^{50}$.

The diagnosis of PSP-CBD requires at least three of the five following features: axial or symmetric limb rigidity; postural instability or falls; urinary incontinence; behavioral changes; supranuclear vertical gaze palsy or decreased velocity of vertical saccades ${ }^{2}$.

\section{Differential diagnosis}

A wide variety of CBD differential diagnoses have been reported in the literature. These syndromes usually resemble CBS but have different underlying pathologies. The most frequent pathologic substrates for clinical CBS are CBD (35\%), $\mathrm{AD}(23 \%)$, PSP (13\%), and fronto-temporal dementia with TDP-43 pathology $(13 \%)^{56,58}$.

The overlap between PSP and CBD has become increasingly recognized, as both are unresponsive to levodopa and pathologically confirmed CBD has occasionally been described with symmetrical clinical features $^{56}$. In addition, both also have overlapping characteristics when variant phenotypes are considered ${ }^{57}$. For instance, the PSP-CBD variant of PSP typically does not present with early gait dysfunction and postural instability. Asymmetrical dyspraxia, dystonia and alien limb phenomena are also typical. Although both PSP and CBD can develop oculomotor abnormalities, the specific eye movements usually are distinct: increased saccadic latencies with preserved velocity as well as equally impaired horizontal and vertical plane saccades suggest CBD, while vertical supranuclear gaze palsy preceded by slowness of vertical saccades are more likely related to PSP ${ }^{56}$. Depression and irritability are more frequent in CBD while apathy is predominant in PSP $^{59}$.
Corticobasal degeneration, PSP and fronto-temporal lobar degeneration also overlap when presented as a behavioral syndrome. The differential diagnosis is challenging, as some CBD patients present with exuberant behavioral disturbances and only develop minimal CBS features late in the disease course ${ }^{56}$. Two genes were described as causes of fronto-temporal lobar degeneration presenting as CBD mimics. Mutations in PGRN most commonly present as a CBS phenotype but can also cause behavioral variantfronto-temporal lobar degeneration and progressive nonfluent aphasia syndromes ${ }^{58}$. Mutation in C9ORF72 was also related to the CBS phenotype. The presence of hallucinations and upper motor neuron syndrome may help to differentiate fronto-temporal lobar degeneration from CBD in these cases. Another important differential diagnosis to be considered is primary progressive aphasia, particularly the nonfluent type, which is most frequently associated with tau-positive pathology ${ }^{56}$. Progressive nonfluent aphasia is part of the spectrum of tauopathies, such as fronto-temporal dementia, PSP and CBD, and manifests with difficulties in sentence comprehension, with equal impairment of aphasia and apraxia of speech when compared with progressive nonfluent aphasia-PSP ${ }^{51}$. Executive dysfunction tests, such as the Delis-Kaplan Executive Function System may help to differentiate between CBS and fronto-temporal dementia patients ${ }^{55}$.

Corticobasal syndrome has also been described in $\mathrm{AD}$ autopsy- confirmed patients. Early age at onset and myoclonus are suggestive of $\mathrm{AD}$ rather than $\mathrm{CBD}$ pathology. Presenilin 1 mutations may manifest as parkinsonism with relatively symmetric myoclonus, apraxia, dystonia, frontal subcortical dementia and, sometimes, seizures ${ }^{60}$. Balint's syndrome, Gerstmann syndrome, visual agnosia or alexia are other possible presentations ${ }^{56}$.

Other gene mutation CBD mimics recently described in the literature were FUS, MAPT, DCTN1, LRRK2, CYP27A1, GBA and PRNP5 .

\section{FINAL REMARKS}

After almost 50 years since its original description, our understanding of CBD has progressed quite substantially and continues to evolve. Over these five decades, its phenomenology grew richer, several aspects of the non-motor manifestations were discovered and explored, pathological findings were delineated, and several facets related to pathophysiology and molecular bases surfaced. The disease's higher cortical features have also been better understood so that cognitive deficits are becoming increasingly important for its diagnosis, especially in the early stages of CBD. Despite these advances, the diagnosis is still challenging due to the overlapping of different tauopathies and the lack of specific biomarkers for each one of them. 
1. Rebeiz JJ, Kolodny EH, Richardson EP. Corticodentatonigral degeneration with neuronal achromasia: a progressive disorder of late adult life. Trans Am Neurol Assoc. 1967;92:23-6.

2. Armstrong MJ, Litvan I, Lang AE, BakTH, Bhatia KP, Borroni B et al. Criteria for the diagnosis of corticobasal degeneration. Neurology. 2013;80(5):496-503. https://doi.org/10.1212/WNL.0b013e31827fofd1

3. Chahine LM, Rebeiz T, Rebeiz JJ, Grossman M, Gross RG. Corticobasal syndrome: five new things. Neurol Clin Pract. 2014;4(4):304-12. https://doi.org/10.1212/CPJ.0000000000000026

4. Litvan I, Agid Y, Goetz C, Jankovic J, Wenning GK, Brandel JP et al. Accuracy of the clinical diagnosis of corticobasal degeneration: a clinicopathologic study. Neurology. 1997;48(1):119-25. https://doi.org/10.1212/WNL.48.1.119

5. Ling H, O'Sullivan SS, Holton JL, Revesz T, Massey LA, Williams DR et al. Does corticobasal degeneration exist? A clinicopathological re-evaluation. Brain. 2010;133(7):2045-57. https://doi.org/10.1093/brain/awq123

6. Josephs KA, Petersen RC, Knopman DS, Boeve BF, Whitwell JL, Duffy JR et al. Clinicopathologic analysis of frontotemporal and corticobasal degenerations and PSP. Neurology. 2006;66(1):41-8. https://doi.org/10.1212/01.wnl.0000191307.69661.c3

7. Ouchi H, Toyoshima Y, Tada M, Oyake M, Aida I, Tomita I et al. Pathology and sensitivity of current clinical criteria in corticobasal syndrome. Mov Disord. 2014;29(2):238-44. https://doi.org/10.1002/mds.25746

8. Osaki Y, Morita Y, Kuwahara T, Miyano I, Doi Y. Prevalence of Parkinson's disease and atypical parkinsonian syndromes in a rural Japanese district. Acta Neurol Scand. 2011;124(3):182-7. https://doi.org/10.1111/j.1600-0404.2010.01442.x

9. Winter Y, Bezdolnyy Y, Katunina E, Avakjan G, Reese JP, Klotsche $J$ et al. Incidence of Parkinson's disease and atypical parkinsonism: russian population-based study. Mov Disord. 2010;25(3):349-56. https://doi.org/10.1002/mds.22966

10. Irwin DJ. Tauopathies as clinicopathological entities. Parkinsonism Relat Disord. 2016;22 Suppl 1:S29-33. https://doi.org/10.1016/j.parkreldis.2015.09.020

11. Alexander SK, Rittman T, Xuereb JH, Bak TH, Hodges JR, Rowe JB. Validation of the new consensus criteria for the diagnosis of corticobasal degeneration.J Neurol Neurosurg Psychiatry. 2014;85(8):925-9. https://doi.org/10.1136/jnnp-2013-307035

12. Munhoz RP, Werneck LC, Teive HA. The differential diagnoses of parkinsonism: findings from a cohort of 1528 patients and a 10 years comparison in tertiary movement disorders clinics. Clin Neurol Neurosurg. 2010;112(5):431-5. https://doi.org/10.1016/j.clineuro.2010.03.003

13. Grijalvo-Perez AM, Litvan I. Corticobasal Degeneration. Semin Neurol. 2014;34(02):160-73. https://doi.org/10.1055/s-0034-1381734

14. Marsili L, Suppa A, Berardelli A, Colosimo C. Therapeutic interventions in parkinsonism: corticobasal degeneration. Parkinsonism Relat Disord. 2016;22 Suppl 1:S96-100. https://doi.org/10.1016/j.parkreldis.2015.09.023

15. Boeve BF. The multiple phenotypes of corticobasal syndrome and corticobasal degeneration: implications for further study. J Mol Neurosci. 2011;45(3):350-3. https://doi.org/10.1007/s12031-011-9624-1

16. Mahapatra RK, Edwards MJ, Schott JM, Bhatia KP. Corticobasal degeneration. Lancet Neurol. 2004;3(12):736-43. https://doi.org/10.1016/S1474-4422(04)00936-6

17. Godeiro-Junior C, Felício AC, Barsottini OG, Aguiar PM, Silva $\mathrm{SM}$, Borges $V$ et al. Clinical features of dystonia in atypical parkinsonism. Arq Neuropsiquiatr. 2008;66(4):800-4. https://doi.org/10.1590/S0004-282X2008000600004

18. Vanek Z, Jankovic J. Dystonia in corticobasal degeneration. Mov Disord. 2001;16(2):252-7. https://doi.org/10.1002/mds.1038
19. Stamelou M, Alonso-Canovas A, Bhatia KP. Dystonia in corticobasal degeneration: a review of the literature on 404 pathologically proven cases. Mov Disord. 2012;27(6):696-702. https://doi.org/10.1002/mds.24992

20. Rinne JO, Lee MS, Thompson PD, Marsden CD. Corticobasal degeneration: A clinical study of 36 cases. Brain. 1994;117(5):1183-96. https://doi.org/10.1093/brain/117.5.1183

21. Thompson PD, Day BL, Rothwell JC, Brown P, Britton TC, Marsden CD. The myoclonus in corticobasal degeneration. Evidence for two forms of cortical reflex myoclonus. Brain. 1994;117 (5):1197-207. https://doi.org/10.1093/brain/117.5.1197

22. Rivaud-Péchoux S, Vidailhet M, Gallouedec G, Litvan I, Gaymard B, Pierrot-Deseilligny C. Longitudinal ocular motor study in corticobasal degeneration and progressive supranuclear palsy. Neurology. 2000;54(5):1029-32. https://doi.org/10.1212/WNL.54.5.1029

23. Armstrong RA. Visual signs and symptoms of corticobasal degeneration. Clin Exp Optom. 2016;99(6):498-506. https://doi.org/10.1111/cxo.12429

24. Boxer AL, Garbutt S, Seeley WW, Jafari A, Heuer HW, Mirsky J et al. Saccade abnormalities in autopsy-confirmed frontotemporal lobar degeneration and Alzheimer's disease. Arch Neurol. 2012;69(4):509-17. https://doi.org/10.1001/archneurol.2011.1021

25. Hassan A, Josephs KA. Alien hand syndrome. Curr Neurol Neurosci Rep. 2016;16(8):73. https://doi.org/10.1007/s11910-016-0676-z

26. Kovacs GG. Invited review: neuropathology of tauopathies: principles and practice. Neuropathol Appl Neurobiol. 2015;41(1):3-23. https://doi.org/10.1111/nan.12208

27. Ishizawa K, Dickson DW. Microglial activation parallels system degeneration in progressive supranuclear palsy and corticobasal degeneration. J Neuropathol Exp Neurol. 2001;60(6):647-57. https://doi.org/10.1093/jnen/60.6.647

28. Kouri N, Whitwell JL, Josephs KA, Rademakers R, Dickson DW. Corticobasal degeneration: a pathologically distinct 4R tauopathy. Nat Rev Neurol. 2011;7(5):263-72. https://doi.org/10.1038/nrneurol.2011.43

29. Dickson DW, Bergeron C, Chin SS, Duyckaerts C, Horoupian D, Ikeda K, et al. Office of rare diseases neuropathologic criteria for corticobasal degeneration. J Neuropathol Exp Neurol. 2002;61(11):935-46. https://doi.org/10.1093/jnen/61.11.935

30. Yoshida M. Astrocytic inclusions in progressive supranuclear palsy and corticobasal degeneration. Neuropathology. 2014;34(6):555-70. https://doi.org/10.1111/neup.12143

31. Houlden H, Baker M, Morris HR, MacDonald N, Pickering-Brown S, Adamson $\mathrm{J}$ et al. Corticobasal degeneration and progressive supranuclear palsy share a common tau haplotype. Neurology. 2001;56(12):1702-6. https://doi.org/10.1212/WNL.56.12.1702

32. Kouri N, Ross OA, Dombroski B, Younkin CS, Serie DJ, Soto-Ortolaza A et al. Genome-wide association study of corticobasal degeneration identifies risk variants shared with progressive supranuclear palsy. Nat Commun. 2015;6:7247. https://doi.org/10.1038/ncomms8247

33. Fekete R, Bainbridge M, Baizabal-Carvallo JF, Rivera A, Miller B, Du P et al. Exome sequencing in familial corticobasal degeneration. Parkinsonism Relat Disord. 2013;19(11):1049-52. https://doi.org/10.1016/j.parkreldis.2013.06.016

34. Zhang Y, Walter R, Ng P, Luong PN, Dutt S, Heuer H et al. Progression of microstructural degeneration in progressive supranuclear palsy and corticobasal syndrome: a longitudinal diffusion tensor imaging study. PLoS One. 2016;11(6):e0157218. https://doi.org/10.1371/journal.pone.0157218

35. Josephs KA, Whitwell JL, Dickson DW, Boeve BF, Knopman DS, Petersen RC et al. Voxel-based morphometry in autopsy proven PSP and CBD. Neurobiol Aging. 2008;29(2):280-9. https://doi.org/10.1016/j.neurobiolaging.2006.09.019 
36. Gröschel K, Hauser TK, Luft A, Patronas N, Dichgans J, Litvan I et al. Magnetic resonance imaging-based volumetry differentiates progressive supranuclear palsy from corticobasal degeneration. Neuroimage. 2004;21(2):714-24. https://doi.org/10.1016/j.neuroimage.2003.09.070

37. Whitwell JL, Jack CR, Parisi JE, Knopman DS, Boeve BF, Petersen RC et al. Rates of cerebral atrophy differ in different degenerative pathologies. Brain. 2007;130(4):1148-58. https://doi.org/10.1093/brain/awm021

38. Borroni B, Garibotto V, Agosti C, Brambati SM, Bellelli G, Gasparotti R et al. White matter changes in corticobasal degeneration syndrome and correlation with limb apraxia. Arch Neurol. 2008;65(6):796-801. https://doi.org/10.1001/archneur.65.6.796

39. Sajjadi SA, Acosta-Cabronero J, Patterson K, Diaz-de-Grenu LZ, Williams GB, Nestor PJ. Diffusion tensor magnetic resonance imaging for single subject diagnosis in neurodegenerative diseases. Brain. 2013 ;136(7):2253-61. https://doi.org/10.1093/brain/awt118

40. Niethammer M, Tang CC, Feigin A, Allen PJ, Heinen L, Hellwig S et al. A disease-specific metabolic brain network associated with corticobasal degeneration. Brain. 2014;137(11):3036-46. https://doi.org/10.1093/brain/awu256

41. Zalewski N, Botha H, Whitwell JL, Lowe V, Dickson DW, Josephs KA. FDG-PET in pathologically confirmed spontaneous 4R-tauopathy variants. J Neurol. 2014;261(4):710-6. https://doi.org/10.1007/s00415-014-7256-4

42. Pirker S, Perju-Dumbrava L, Kovacs GG, Traub-Weidinger T, Asenbaum S, Pirker W. Dopamine D2 receptor SPECT in corticobasal syndrome and autopsy-confirmed corticobasal degeneration. Parkinsonism Relat Disord. 2013;19(2):222-6. https://doi.org/10.1016/j.parkreldis.2012.10.010

43. Pirker S, Perju-Dumbrava L, Kovacs GG, Traub-Weidinger T, Pirker W. Progressive dopamine transporter binding loss in autopsy-confirmed corticobasal degeneration. J Parkinsons Dis. 2015;5(4):907-12. https://doi.org/10.3233/JPD-150625

44. Maruyama M, Shimada H, Suhara T, Shinotoh H, Ji B, Maeda $\mathrm{J}$ et al. Imaging of tau pathology in a tauopathy mouse model and in Alzheimer patients compared to normal controls. Neuron. 2013;79(6):1094-108. https://doi.org/10.1016/j.neuron.2013.07.037

45. Josephs KA, Whitwell JL, Tacik P, Duffy JR, Senjem ML, Tosakulwong Net al. [18F]AV-1451 tau-PET uptake does correlate with quantitatively measured 4R-tau burden in autopsy-confirmed corticobasal degeneration. Acta Neuropathol. 2016;132(6):931-3. https://doi.org/10.1007/s00401-016-1618-1

46. Wagshal D, Sankaranarayanan S, Guss V, Hall T, Berisha F, Lobach I et al. Divergent CSF $\tau$ alterations in two common tauopathies: Alzheimer's disease and progressive supranuclear palsy.J Neurol Neurosurg Psychiatry. 2015 ;86(3):244-50. https://doi.org/10.1136/jnnp-2014-308004

47. VanVoorst WA, Greenaway MC, Boeve BF, Ivnik RJ, Parisi JE, Eric Ahlskog $\mathrm{J}$ et al. Neuropsychological findings in clinically atypical autopsy confirmed corticobasal degeneration and progressive supranuclear palsy. Parkinsonism Relat Disord. 2008;14(4):376-8. https://doi.org/10.1016/j.parkreldis.2007.09.006

48. Cassidy A. The clinical assessment of apraxia. Pract Neurol. 2016;16(4):317-22. https://doi.org/10.1136/practneurol-2015-001354

49. Leiguarda RC, Marsden CD. Limb apraxias: higher-order disorders of sensorimotor integration. Brain. 2000;123(5):860-79. https://doi.org/10.1093/brain/123.5.860

50. McMonagle P, Blair M, Kertesz A. Corticobasal degeneration and progressive aphasia. Neurology. 2006;67(8):1444-51. https://doi.org/10.1212/01.wnl.0000240215.43492.01

51. Rittman T, Ghosh BC, McColgan P, Breen DP, Evans J, Williams-Gray $\mathrm{CH}$ et al. The Addenbrooke's cognitive examination for the differential diagnosis and longitudinal assessment of patients with parkinsonian disorders. J Neurol Neurosurg Psychiatry. 2013;84(5):544-51. https://doi.org/10.1136/jnnp-2012-303618

52. Mathew R, BakTH, Hodges JR. Screening for cognitive dysfunction in corticobasal syndrome: utility of Addenbrooke's cognitive examination. Dement Geriatr Cogn Disord. 2011;31(4):254-8. https://doi.org/10.1159/000327169

53. Grossman M, Irwin DJ. The mental status examination in patients with suspected dementia. Continuum (Minneap Minn). 2016;22(2 Dementia):385-403. https://doi.org/10.1212/CON.0000000000000298

54. Burrell JR, Hodges, JR, Rowe JB. Cognition in corticobasal syndrome and progressive supranuclear palsy: a review. Mov Disord. 2014;29(5):684-93. https://doi.org/10.1002/mds.25872

55. Huey ED, Goveia EN, Paviol S, Pardini M, Krueger F, Zamboni $G$ et al. Executive dysfunction in frontotemporal dementia and corticobasal syndrome. Neurology. 2009;72(5):453-9. https://doi.org/10.1212/01.wnl.0000341781.39164.26

56. Kouri N, Whitwell JL, Josephs KA, Rademakers R, Dickson DW. Corticobasal degeneration: a pathologically distinct 4R tauopathy. Nat Rev Neurol 2011;7(5):263-72. https://doi.org/10.1038/nrneurol.2011.43

57. Greene JD. Apraxia, agnosias, and higher visual function abnormalities. J Neurol Neurosurg Psychiatry. 2005;76(Suppl 5):v25-34. https://doi.org/10.1136/jnnp.2005.081885

58. Ludolph AC, Kassubek J, Landwehrmeyer BG, Mandelkow E, Mandelkow EM, Burn DJ et al. Tauopathies with parkinsonism: clinical spectrum, neuropathologic basis, biological markers, and treatment options. Eur J Neurol. 2009;16(3):297-309. https://doi.org/10.1111/j.1468-1331.2008.02513.x

59. Cummings JL, Litvan I. Neuropsychiatric aspects of corticobasal degeneration. Adv. Neurol. 2000;82:147-52.

60. Stamelou M, Quinn NP, Bhatia KP. “Atypical” atypical parkinsonism: new genetic conditions presenting with features of progressive supranuclear palsy, corticobasal degeneration, or multiple system atrophy: a diagnostic guide. Mov Disord. 2013;28(9):1184-99. https://doi.org/10.1002/mds.25509 